Теорія Ймовір. та Матем. Статист. Вип. 77, 2007
Theor. Probability and Math. Statist.

No. 77, 2008, Pages 135-146 S 0094-9000(09)00752-2

Article electronically published on January 16, 2009

\title{
A BOUNDED ARBITRAGE STRATEGY FOR A MULTIPERIOD MODEL OF A FINANCIAL MARKET IN DISCRETE TIME
}

UDC 519.21

\author{
YU. S. MISHURA, P. S. SHELYAZHENKO, AND G. M. SHEVCHENKO
}

\begin{abstract}
The notion of $\varepsilon$-arbitrage strategy is introduced for a multiperiod model. A theorem, analogous to the classical first fundamental theorem for a usual arbitrage strategy, is proved for this model. The difference between single-period and multiperiod models is discussed.
\end{abstract}

\section{INTRODUCTION}

The following approach is extensively used to model financial markets. It is assumed that the prices in a financial market change randomly; according to these changes, an investor chooses a strategy and transforms an initial capital $V_{0}$ into a terminal value during the time $T$. The terminal capital is treated as an element of the set of all possible terminal values.

A natural question that arises for such models is whether or not an arbitrage strategy exists. In other words, is it possible for investors to find a riskless strategy allowing them to finish with a positive profit. Conditions for the existence of arbitrage strategies are studied in many papers. It is known for models with discrete time that the existence of an arbitrage strategy is related to the existence of an equivalent martingale measure for the price process. This case is discussed in detail in [1] and [2]. Delbaen and Schachermayer [3] consider arbitrary continuous semimartingales in continuous time. It is proved in [3] that if there is no arbitrage strategy, then an absolutely continuous locally martingale measure exists. Later, Kabanov and Stricker 4] generalized this result and dropped the assumption of the continuity of semimartingales. The classical notion of arbitrage strategy is discussed in the books [5, 6.

The notion of arbitrage strategy is extended in the paper [7]. In contrast to the case of large markets, small financial markets may have an analogue of arbitrage strategy for which the profit is bounded in a certain sense (note that the market itself is bounded). Such an arbitrage strategy is called a bounded or an $\varepsilon$-arbitrage strategy. The mathematical tools used to study such strategies are different from the classical techniques, since the markets without $\varepsilon$-arbitrage strategies may have a classical arbitrage strategy.

The basic notions of $\varepsilon$-arbitrage strategies are introduced in [7. An analogue of the first fundamental theorem of financial markets is also proved in [7] for the case of a bounded arbitrage strategy and for a single-period model with nonrandom initial data.

In this paper, we continue developing results similar to the classical arbitrage theory for multiperiod models of financial markets in discrete time. We prove an analogue of

2000 Mathematics Subject Classification. Primary 91B28.

Key words and phrases. Arbitrage strategy, $\varepsilon$-arbitrage strategy, financial market, multiperiod model, self-financing strategy. 
the classical Dalang-Morton-Willinger theorem (see [2, 8]) for the case of a bounded arbitrage strategy and for a multiperiod model. This result provides several conditions that are equivalent to the nonexistence of a bounded arbitrage strategy in the case of multiperiod models of financial markets.

\section{MAin NOTATION}

We introduce the following notation. Let $(\Omega, \mathbb{F}, \mathrm{P})$ be a complete probability space. Consider a market with a single riskless asset and $d$ risky assets. Denote by

$$
\bar{\pi}=\left(\pi_{0}, \pi\right)=\left(\pi_{0}, \pi_{1}, \ldots, \pi_{d}\right) \in \mathbb{R}^{d+1}, \quad d \geq 1,
$$

the nonrandom vector of initial prices. The random vector of prices at the moment $t=1$ is denoted by

$$
\bar{S}=\left(S^{0}, S\right)=\left(S^{0}, S^{1}, \ldots, S^{d}\right) .
$$

Without loss of generality we assume that $\pi_{0}=1$ and $S^{0}=1+r$, where $r>0$ is the nonrandom interest rate.

The investor portfolio is denoted by $\bar{\xi}=\left(\xi^{0}, \xi\right)=\left(\xi^{0}, \xi^{1}, \ldots, \xi^{d}\right) \in \mathbb{R}^{d+1}$. Then the wealth of an investor at the moments $t=0$ and $t=1$ is equal to $\bar{\xi} \cdot \bar{\pi}$ and $\bar{\xi} \cdot \bar{S}$, respectively.

Definition 2.1. We say that a financial market admits an $\varepsilon$-arbitrage strategy if there exists $\bar{\xi} \in \mathbb{R}^{d+1}$ such that $\|\xi\|_{1}:=\sum_{i=1}^{d}\left|\xi_{i}\right| \leq 1$ and $\bar{\xi} \cdot \bar{\pi} \leq-\varepsilon$, while $\bar{\xi} \cdot \bar{S} \geq 0$ P-almost surely and $\mathrm{P}\{\bar{\xi} \cdot \bar{S}>0\}>0$.

Theorem 2.2 ([7]). The following conditions are equivalent:

1. There is no $\varepsilon$-arbitrage strategy in the financial market.

2. There exists a measure $\mathrm{P}^{*}$ equivalent to $\mathrm{P}$ and such that

$$
\left|\pi_{i}-\mathrm{E}_{\mathrm{P}^{*}} \frac{S^{i}}{1+r}\right| \leq \varepsilon
$$

for all $i=1, \ldots, d$. Moreover, the Radon-Nikodym derivative is bounded, that is $d \mathrm{P}^{*} / d \mathrm{P} \leq C$ for some $C>0$.

This result is an analogue of the first fundamental theorem for a single-period model. An essential restriction of this result is that the initial data are nonrandom. This does not allow us to apply it subsequently for each period of a multiperiod model, since the price vector is random at the beginning of every period.

Below we provide a similar result but for a more general model.

\section{MAIN THEOREM FOR A SINGLE-PERIOD MODEL WITH RANDOM INITIAL DATA}

We introduce some new notation. Let $(\Omega, \mathbb{F}, \mathrm{P})$ be a complete probability space equipped with a filtration $\left(\mathbb{F}_{t}\right)_{t=0, \ldots, T}$ such that $\mathbb{F}_{T}=\mathbb{F}$. Let $\bar{S}=\left(\bar{S}_{t}\right), t=0, \ldots, T$, be an $(d+1)$-dimensional price process (price vector) adapted to $\mathbb{F}_{t}$. Without loss of generality, we assume that $S_{0}^{0}=1$ and $S_{t}^{0}=(1+r)^{t}$, where $r>0$ is a nonrandom interest rate.

A $(d+1)$-dimensional process $\bar{\xi}=\left(\xi^{0}, \xi\right)=\left(\xi_{t}^{0}, \xi_{t}^{1}, \ldots, \xi_{t}^{d}\right)$ is called a strategy if it is adapted to $\mathbb{F}_{t}$.

A strategy $\bar{\xi}=\left(\bar{\xi}_{t}\right), t=0, \ldots, T-1$, is called self-financing if

$$
\bar{\xi}_{t-1} \cdot \bar{S}_{t}=\bar{\xi}_{t} \cdot \bar{S}_{t}
$$

for all $t=1, \ldots, T-1$.

We define the discounted price process with respect to $S^{0}$ by

$$
X_{t}^{i}:=\frac{S_{t}^{i}}{S_{t}^{0}}, \quad t=0, \ldots, T, i=0, \ldots, d .
$$


Put $\bar{X}_{t}:=\left(X_{t}^{0}, X_{t}\right)=\left(X_{t}^{0}, X_{t}^{1}, \ldots, X_{t}^{d}\right)$.

A process

$$
V_{0}:=\bar{\xi}_{0} \cdot \bar{X}_{0}, \quad V_{t}:=\bar{\xi}_{t-1} \cdot \bar{X}_{t}, \quad t=1, \ldots, T,
$$

is called the discounted capital process related to a strategy $\bar{\xi}_{t}$.

The following definition is equivalent to Definition 2.1. however, we apply it to the model under consideration.

Definition 3.1. We say that a multiperiod market admits an $\varepsilon$-arbitrage strategy if there exists a self-financing strategy $\bar{\xi}=\left(\overline{\xi_{t}}\right)$ bounded in the sense that

$$
\left\|\xi_{t}\right\|_{1}:=\left\|\sum_{i=1}^{d}\left|\xi_{t}^{i}\right|\right\|_{L_{\infty}(\Omega)} \leq 1 \text { for all } t=0, \ldots, T-1,
$$

and such that the capital process related to $\bar{\xi}$ satisfies the following conditions:

$$
V_{0} \leq-\varepsilon, \quad \mathrm{P}\left\{V_{T} \geq 0\right\}=1, \quad \mathrm{P}\left\{V_{T}>0\right\}>0 .
$$

Remark 3.2. Condition (1) is a generalization of the boundedness condition with respect to the norm $\|\cdot\|_{1}$ for a single-period model written for all periods. Since $\left\|\xi_{t}\right\|_{1}$ is a random variable for all $t$, we use the norm $\|\cdot\|_{L_{\infty}(\Omega)}$ (the essential supremum with respect to $\omega \in \Omega$ ).

Consider the case of $T=1$, that is, a single-period model with random initial data. Put $\triangle X=X_{1}-X_{0}$ and $\|x\|_{\infty}=\max _{i}\left|x^{i}\right|$.

The following result is proved similarly to the corresponding result in [7].

Lemma 3.3. The following statements are equivalent:

1. The financial market admits an $\varepsilon$-arbitrage strategy.

2. There exists an $\mathbb{F}_{0}$-dimensional vector $\bar{\xi}$ such that

$$
\left\|\sum_{i=1}^{d}\left|\xi^{i}\right|\right\|_{L_{\infty}(\Omega)} \leq 1
$$

and moreover $\xi \cdot\left(X_{1}-X_{0}\right) \geq \varepsilon \mathrm{P}$-almost surely and $\mathrm{P}\left\{\xi \cdot\left(X_{1}-X_{0}\right)>\varepsilon\right\}>0$.

Now we turn to main results of this sections.

Lemma 3.4. Assume that there exists a measure $\mathrm{P}^{*}$ equivalent to $\mathrm{P}$ and such that

$$
\mathrm{E}_{\mathrm{P}^{*}}\left[\left\|\mathrm{E}_{\mathrm{P}^{*}}\left[\triangle X / \mathbb{F}_{0}\right]\right\|_{\infty}\right] \leq \varepsilon .
$$

Then there is no $\varepsilon$-arbitrage strategy in the financial market.

Proof. Assume that there exists a strategy $\xi$ that generates an $\varepsilon$-arbitrage strategy and that is bounded in the sense of definition (1). According to Lemma 3.3.

$$
\xi \cdot \triangle X \geq \varepsilon, \quad \mathrm{P}\{\xi \cdot \triangle X>\varepsilon\}>0 .
$$

Thus $\mathrm{P}^{*}\{\xi \cdot \triangle X>\varepsilon\}>0$, since the measures are equivalent. This implies $\mathrm{E}_{\mathrm{P} *}[\xi \cdot \Delta X]>\varepsilon$. On the other hand,

$$
\begin{aligned}
\mathrm{E}_{\mathrm{P}^{*}}[\xi \cdot \Delta X] & =\mathrm{E}_{\mathrm{P}^{*}}\left[\mathrm{E}_{\mathrm{P}^{*}}\left[\xi \cdot \Delta X / \mathbb{F}_{0}\right]\right] \\
& \leq \mathrm{E}_{\mathrm{P}^{*}}\left[\|\xi\|_{1}\left\|\mathrm{E}_{\mathrm{P}^{*}}\left[\triangle X / \mathbb{F}_{0}\right]\right\|_{\infty}\right] \leq \mathrm{E}_{\mathrm{P}^{*}}\left[\left\|\mathrm{E}_{\mathrm{P}^{*}}\left[\triangle X / \mathbb{F}_{0}\right]\right\|_{\infty}\right] \leq \varepsilon .
\end{aligned}
$$

This contradiction means that the above assumption of the existence of a strategy $\xi$ is incorrect, whence we conclude that an $\varepsilon$-arbitrage strategy does not exist. 
Theorem 3.5. The following conditions are equivalent for the model under consideration:

1. The financial market does not admit an $\varepsilon$-arbitrage strategy.

2. There exists a measure $\mathrm{P}^{*}$ equivalent to $\mathrm{P}$ and such that $d \mathrm{P}^{*} / d \mathrm{P} \leq C$ for some $C>0$ and

$$
\mathrm{E}_{\mathrm{P} *}\left[\left\|\mathrm{E}_{\mathrm{P} *}\left[\triangle X / \mathbb{F}_{0}\right]\right\|_{\infty}\right] \leq \varepsilon .
$$

Proof. $[2 \Rightarrow 1]$ This part is a direct consequence of Lemma 3.4 .

$[1 \Rightarrow 2]$ Note that the additional assumption

$$
\mathrm{E}\left[X_{t}^{i}\right]<\infty, \quad i=1, \ldots, d, t=0,1,
$$

does not restrict generality. Indeed, we introduce the probability measure $\tilde{P}$ as follows:

$$
\frac{d \tilde{\mathrm{P}}}{d \mathrm{P}}:=c\left(1+\sum_{i=1}^{d} \sum_{t=0}^{1} X_{t}^{i}\right)^{-1}
$$

where $c$ is such that the expectation of the right hand side is equal to 1 . It is clear that condition (3) holds for $\tilde{P}$. Condition 1 of Theorem 3.5 holds for the measure $P$ if and only if it holds for the equivalent measure $\tilde{P}$. If the measure $\mathrm{P}^{*}$ is such that the density $d \mathrm{P}^{*} / d \tilde{\mathrm{P}}$ is bounded, then the same holds for the density

$$
\frac{d \mathrm{P}^{*}}{d \mathrm{P}}=\frac{d \mathrm{P}^{*}}{d \tilde{\mathrm{P}}} \frac{d \tilde{\mathrm{P}}}{d \mathrm{P}} .
$$

Thus the implication $1 \Rightarrow 2$ holds for $\mathrm{P}$ if and only if it holds for $\tilde{P}$. This proves that one can additionally assume that (3) holds.

Put $Y:=\triangle X$. The rest of the proof follows the lines of that in $[2$.

First we prove that an equivalent measure with continuous density exists if the model with nonrandom initial data does not admit an $\varepsilon$-arbitrage strategy. Denote by $\overline{\mathbb{R}}^{d}$ the single-point compactification of the space $\mathbb{R}^{d}$. For a vector $\alpha \in \mathbb{R}^{d}$, put $H_{\alpha \varpi}^{\varepsilon}=$ $\{x: \alpha \cdot x \varpi \varepsilon\}$, where the symbol $\varpi$ stands for one of the signs $\{\leq,<,=,>, \geq\}$.

Lemma 3.6. Let $Y \in \mathbb{R}^{d}$ be a random vector with distribution $\mathrm{P}(d x)$. Assume that there is no $\varepsilon$-arbitrage strategy for $Y$; that is, if $\mathrm{P}\left(H_{\alpha \geq}^{\varepsilon}\right)=1$ for $\alpha \in \mathbb{R}^{d},\|\alpha\|_{1} \leq 1$, then $\mathrm{P}\left(H_{\alpha>}^{\varepsilon}\right)=0$. Then there exists a positive function $g \in C\left(\overline{\mathbb{R}}^{d}\right)$ such that $\mathrm{E}[g(Y)]=1$ and $\|\mathrm{E}[Y g(Y)]\|_{\infty} \leq \varepsilon$.

Proof. Let

$$
F=\left\{g: g \in C\left(\overline{\mathbb{R}}^{d}\right), g>0, \mathrm{E}[g(Y)]=1\right\} .
$$

Then $F \neq \varnothing$, since $1 \in F$. Consider a nonempty convex set

$$
C=\{\mathrm{E}[Y g(Y)]: g \in F\} .
$$

Assume that $C \cap[-\varepsilon, \varepsilon]^{d}=\varnothing$. Then the sets $C$ and $[-\varepsilon, \varepsilon]^{d}$ can be separated. In other words, there exists a vector $\alpha \in \mathbb{R}^{d}$ such that $C \subset H_{\alpha>}^{\varepsilon}$ and $[-\varepsilon, \varepsilon]^{d} \subset H_{\alpha \leq}^{\varepsilon}$. The second inclusion is equivalent to $\|\alpha\|_{1} \leq 1$. Assume that $\mathrm{P}\left(H_{\alpha<}^{\varepsilon}\right)>0$. Consider a sequence $f_{n} \in C\left(\overline{\mathbb{R}}^{d}\right)$ such that $f_{n} \in(0,1]$ and $f_{n} \rightarrow \mathbb{1}_{H_{\alpha<}^{\varepsilon}}$. Then $\mathrm{E}\left[Y f_{n}(Y)\right] \rightarrow \mathrm{E}\left[Y \mathbb{1}_{H_{\alpha<}^{\varepsilon}}\right]$ and $\mathrm{E}\left[f_{n}(Y)\right] \rightarrow \mathrm{P}\left(H_{\alpha<<}^{\varepsilon}\right)$. In particular, $\mathrm{E}\left[f_{n}(Y)\right]>0$ for sufficiently large $n$. Then

$$
\begin{aligned}
\varepsilon & \leq \lim _{n \rightarrow \infty} \alpha \cdot \mathrm{E}\left[Y g_{n}(Y)\right] \\
& =\alpha \cdot \mathrm{E}\left[Y \mathbb{1}_{H_{\alpha<}^{\varepsilon}(Y)}\right] / \mathrm{P}\left(H_{\alpha<}^{\varepsilon}\right)=\mathrm{E}\left[\alpha \cdot Y \mathbb{1}_{H_{\alpha<}^{\varepsilon}}(Y)\right] / \mathrm{P}\left(H_{\alpha<}^{\varepsilon}\right)<\varepsilon,
\end{aligned}
$$

where $g_{n}=f_{n} / \mathrm{E}\left[f_{n}(Y)\right] \in F$.

This contradiction means that $\mathrm{P}\left(H_{\alpha<}^{\varepsilon}\right)=0$, that is, $\mathrm{P}\left(H_{\alpha \geq}^{\varepsilon}\right)=1$. Thus $\mathrm{P}\left(H_{\alpha=}^{\varepsilon}\right)=1$ and $\|\alpha\|_{1}=1$ if an $\varepsilon$-arbitrage strategy does not exist. 
Among vectors $\zeta$ such that $\mathrm{P}\left(H_{\zeta=}^{\varepsilon}\right)=1$ we choose a vector with the minimum number of zero coordinates. Suppose it coincides with $\alpha$. Without loss of generality we assume that $\alpha_{i} \neq 0, i \leq k$, and $\alpha^{i}=0, i \geq k+1$, for some $1 \leq k \leq d$. Consider the set

$$
K=\left\{x \in \mathbb{R}^{d}: x^{i}=\varepsilon \operatorname{sgn} \alpha^{i}, i \leq k,\left|x_{i}\right| \leq \varepsilon, i \geq k+1\right\} .
$$

Assume that $K \cap C=\varnothing$. The sets $K$ and $C$ can be strictly separated; that is, there exists a vector $\beta$ such that $K \subset H_{\beta \leq}^{\varepsilon}, C \subset H_{\beta \geq}^{\varepsilon}$, and $\inf _{K} \beta \cdot x<\sup _{C} \beta \cdot x$. Since $K \subset H_{\beta \leq}^{\varepsilon}$, we have

$$
\sum_{i=1}^{k} \beta^{i} \operatorname{sgn} \alpha^{i}+\sum_{i=k+1}^{d}\left|\beta^{i}\right| \leq 1
$$

Define the strategy $\gamma$ by

$$
\gamma=\alpha+\delta \beta, \quad \text { where } \delta=\min _{1 \leq i \leq k}\left|\alpha^{i}\right| /\left(\max _{i}\left|\beta^{i}\right|+1\right)
$$

Then $\|\gamma\|_{1} \leq 1+\delta$. Putting $\eta=\gamma /(1+\delta)$, we get $C \subset H_{\eta \geq}^{\varepsilon}$ and $\|\eta\|_{1} \leq 1$. Reasoning as above we conclude that $\mathrm{P}\left(H_{\eta=}^{\varepsilon}\right)=1$ and $\|\eta\|_{1}=1$. Since $\alpha$ has the minimum number of zero components, we obtain $\eta^{i}=0, i \geq k+1$. This implies

$$
\eta \cdot x=\sum_{i=1}^{k} \varepsilon\left|\eta_{i}\right|=\varepsilon\|\eta\|_{1}=\varepsilon, \quad x \in K .
$$

Now $\eta \cdot x=\varepsilon, x \in C$, in view of $\mathrm{P}\left(H_{\eta=}^{\varepsilon}\right)=1$. Similarly $\alpha \cdot x=\varepsilon, x \in K \cup C$. Then $\inf _{K} \beta \cdot x=\sup _{C} \beta \cdot x=\varepsilon$ contradicting the strict separation.

Lemma 3.6 allows us to prove the existence of an equivalent martingale measure for the case of random initial data similarly to the paper [2]. We point out only the main steps of the proof and omit the details that can be found in [2].

By $P(\omega, d x)$ we denote the regular conditional distribution of $Y$ given $\mathbb{F}_{0}$. We treat $C\left(\overline{\mathbb{R}}^{d}\right)$ as a normed space equipped with the norm $\|g\|_{\text {sup }}=\max _{\overline{\mathbb{R}}^{d}}|g|$. Let $c(\omega)=$ $\sup _{\|y\|_{1}=1} \operatorname{essinf}_{x \in \mathbb{R}^{d}} x \cdot y$, where the essential supremum is considered with respect to the measure $P(\omega, \cdot)$. We prove that the function $c$ is $\mathbb{F}_{0}$-bounded. It is clear that

$$
\begin{aligned}
\{c(\omega) & >K\} \\
= & \left\{\exists r \in(K, \infty) \cap \mathbb{Q} \forall t \in(0,1) \cap \mathbb{Q} \exists z \in \mathbb{Q}^{d},\|z\|_{1}=1: P\left(\omega, H_{z>}^{r}\right)>t\right\} .
\end{aligned}
$$

Indeed, if $c(\omega)>K$, then there are $r>K, r \in \mathbb{Q}$, and $y \in \mathbb{R}^{d},\|y\|_{1}=1$, such that $P\left(\omega, H_{y>}^{r}\right)=1$. Let $z_{n} \in \mathbb{Q}^{d}$ be such that $\left\|z_{n}\right\|_{1}=1$ and $z_{n} \rightarrow y$ as $n \rightarrow \infty$. If $x \in H_{y>}^{r}$, then $x \cdot y>r$. Hence for all sufficiently large $n$ we have $x \cdot z_{n}>r$ implying $x \in H_{z_{n}>}^{r}$. Thus $H_{y>}^{r} \subset \underline{\lim } H_{z_{n}>}^{r}$, whence $\underline{\lim \mathrm{P}}\left(H_{z_{n}>}^{r}\right) \geq \mathrm{P}\left(H_{y>}^{r}\right)=1$. On the other hand, if $\omega$ belongs to the right hand side of (5), then there is $r>K$ for which one can choose a vector $z_{n} \in \mathbb{Q}^{d}$ such that $\left\|z_{n}\right\|_{1}=1$ and $P\left(\omega, H_{z_{n}>}^{r}\right)>1-1 / n$ for any $n>1$.

The sequence $\left\{z_{n}\right\}$ contains a convergent subsequence. Without loss of generality we assume that the sequence itself is convergent, that is, $z_{n} \rightarrow y$ as $n \rightarrow \infty$. If $x \in H_{z_{n}>}^{r}$ for infinitely many $n$, that is, $x \cdot z_{n}>r$, then $x \cdot y \geq r$, whence $x \in H_{y \geq}^{r}$. Thus $\varlimsup H_{z_{n}>}^{r} \subset H_{y \geq}^{r}$ and $1=\varlimsup \lim P\left(\omega, H_{z_{n}>}^{r}\right) \leq P\left(\omega, H_{y \geq}^{r}\right)$. This implies that $c(\omega)>K$.

It remains to note that the right hand side of (5) is produced by a countable number of operations using the sets $\left\{\omega: \mathrm{P}\left(\omega, H_{z>}^{r}\right)>t\right\}$ that are measurable by the property of a regular conditional probability. Moreover,

$$
c(\omega) \leq \underset{\Omega}{\operatorname{essinf}}\left\|\mathrm{E}\left[Y(\omega) / \mathbb{F}_{0}\right]\right\|_{\infty} \leq d \mathrm{E}\left[\|Y\| / \mathbb{F}_{0}\right],
$$

whence $\mathrm{E}[c(\omega)]<\infty$.

Further we consider two cases. 
Case 1. $c(\omega) \leq \varepsilon$ almost surely.

Consider the set

$\mathfrak{H}=\left\{(\omega, g) \in \Omega \times C\left(\overline{\mathbb{R}}^{d}\right): g \geq 0, \int_{\mathbb{R}^{d}} g(x) P(\omega, d x)=1, \int_{\mathbb{R}^{d}} x g(x) P(\omega, d x) \in[-\varepsilon, \varepsilon]^{d}\right\}$.

It belongs to $\mathbb{F}_{0} \otimes \mathcal{B}\left(C\left(\overline{\mathbb{R}}^{d}\right)\right)$. Since there is no $\varepsilon$-arbitrage strategy, it follows that, for almost all $\omega$, there is no $\varepsilon$-arbitrage strategy for a random vector with distribution $P(\omega, d x)$. Lemma 3.6 implies that, for almost all $\omega$, there exists $g_{\omega} \in C\left(\overline{\mathbb{R}}^{d}\right)$ such that $\left(\omega, g_{\omega}\right) \in \mathfrak{H}$ or, in other words, the projection of $\mathfrak{H}$ to $\Omega$ has full probability. Since $C\left(\overline{\mathbb{R}}^{d}\right)$ is a complete separable space, the measurable selection theorem implies that there exists an $\mathbb{F}_{0}$-measurable function $G: \Omega \rightarrow C\left(\overline{\mathbb{R}}^{d}\right)$ such that $(\omega, G(\omega)) \in \mathfrak{H}$ almost surely. The mapping $(\omega, x) \rightarrow G(\omega, x):=G(\omega)(x)$ is $\mathbb{F}_{0} \otimes \mathcal{B}\left(\mathbb{R}^{d}\right)$-measurable as a superposition of measurable mappings. Thus

$$
D(\omega):=M(\omega) G(\omega, Y(\omega)),
$$

where $M(\omega)=1 /(1+K(\omega))$ and $K(\omega)=\|G(\omega, \cdot)\|_{\text {sup }}$, is $\mathbb{F}_{1}$-measurable.

Note that

$$
\mathrm{E}[G(\omega, Y(\omega))]=\mathrm{E}\left[\mathrm{E}\left[G(\omega, Y(\omega)) / \mathbb{F}_{0}\right]\right]=\mathrm{E}\left[\int_{\mathbb{R}^{d}} g(\omega, x) P(\omega, d x)\right]=1
$$

and similarly

$$
M(\omega)=\mathrm{E}\left[D(\omega) / \mathbb{F}_{0}\right]
$$

Now let $d \mathrm{P}^{*}(\omega)=M D(\omega) d \mathrm{P}(\omega)$, where $M=1 / \mathrm{E}[D(\omega)]$. Then

$$
\begin{aligned}
\mathrm{E}_{P^{*}}\left[\left\|\mathrm{E}_{P^{*}}\left[Y / \mathbb{F}_{0}\right]\right\|_{\infty}\right] & =M \mathrm{E}\left[D(\omega)\left\|\mathrm{E}\left[Y(\omega) D(\omega) / \mathbb{F}_{0}\right] / \mathrm{E}\left[D(\omega) / \mathbb{F}_{0}\right]\right\|_{\infty}\right] \\
& =M \mathrm{E}\left[D(\omega)\left\|\mathrm{E}\left[Y(\omega) G(\omega, Y(\omega)) / \mathbb{F}_{0}\right]\right\|_{\infty}\right] \\
& =M \mathrm{E}\left[D(\omega)\left\|\int_{\mathbb{R}^{d}} x G(\omega, x) P(\omega, d x)\right\|_{\infty}\right] \leq \varepsilon .
\end{aligned}
$$

Case 2. $\mathrm{P}(c(\omega)>\varepsilon)>0$.

Since there is no $\varepsilon$-arbitrage strategy, $\mathrm{P}(c(\omega)<\varepsilon)>0$. Then $\mathrm{P}(A(\delta))>0$ for some $\delta>0$, where $A(\delta)=\{c(\omega)+\delta<\varepsilon\}$. On the other hand, there is no $(c(\omega)+\delta)$-arbitrage strategy for any $\omega$ and for the distribution $P(\omega, d x)$ (this follows from the definition of $c(\omega))$. As before, we prove the existence of a measurable function

$$
G: \Omega \rightarrow C\left(\overline{\mathbb{R}}^{d}\right)
$$

such that $\mathrm{E}\left[G(\omega, Y(\omega)) / \mathbb{F}_{0}\right]=1$, whence

$$
\left\|\mathrm{E}\left[Y(\omega) G(\omega, Y(\omega)) / \mathbb{F}_{0}\right]\right\| \leq c(\omega)+\delta .
$$

Let $M(\omega)$ be the same as above and $M_{1}=1 / \mathrm{E}\left[M(\omega) \mathbb{1}_{A(\delta)}\right], M_{2}=1 / \mathrm{E}\left[M(\omega) \mathbb{1}_{A^{c}(\delta)}\right]$. Put

$$
\rho_{\theta}(\omega)=M(\omega)\left(\theta M_{1} \mathbb{1}_{A(\delta)}+(1-\theta) M_{2} \mathbb{1}_{A^{c}(\delta)}\right) G(\omega, Y(\omega)), \quad \theta \in(0,1) .
$$

The function $\rho_{\theta}$ is positive and bounded. Since $A(\delta) \in \mathbb{F}_{0}$, we pass to the conditional expectations and obtain $\mathrm{E}\left[\rho_{\theta}\right]=1$ and

$$
\mathrm{E}\left[\rho_{\theta}(c(\omega)+\delta)\right]=\theta \mathrm{E}\left[(c(\omega)+\delta) \mathbb{1}_{A(\delta)}\right]+(1-\theta) \mathrm{E}\left[(c(\omega)+\delta) \mathbb{1}_{A^{c}(\delta)}\right] .
$$

Note that the expectation is finite, since $\rho$ is bounded and $c$ is integrable. The latter expression approaches $\mathrm{E}\left[(c(\omega)+\delta) \mathbb{1}_{A(\delta)}\right]<\varepsilon$ as $\theta \rightarrow 0$. Hence there exists $\theta_{0}>0$ such that $\mathrm{E}\left[\rho_{\theta_{0}}(c(\omega)+\delta)\right]<\varepsilon$. Let

$$
d \mathrm{P}^{*}=\rho_{\theta_{0}}(\omega) d \mathrm{P} .
$$


Then

$$
\begin{aligned}
\mathrm{E}_{P^{*}}\left[\left\|\mathrm{E}_{P^{*}}\left[Y / \mathbb{F}_{0}\right]\right\|_{\infty}\right] & =\mathrm{E}_{P^{*}}\left[\left\|\mathrm{E}\left[Y(\omega) G(\omega, Y(\omega)) / \mathbb{F}_{0}\right]\right\|_{\infty}\right] \\
& \leq \mathrm{E}\left[\rho_{\theta_{0}}(c(\omega)+\delta)\right]<\varepsilon .
\end{aligned}
$$

To prove the no-arbitrage property for a multiperiod model, we need an auxiliary result. Let $\|\cdot\|_{\sim}$ be a norm in $\mathbb{R}^{d}$ and let $\|\cdot\|_{\sim}^{*}$ be its conjugate norm. An $\mathbb{F}_{0}$-measurable strategy $\xi \in \mathbb{R}^{d}$ is called an $\varepsilon \sim$-arbitrage strategy for a random vector $X \in \mathbb{R}^{d}$ in a single-period model if $\|\xi\|_{\sim} \leq 1, \xi \cdot X \geq \varepsilon$ almost surely, and $\mathrm{P}(\xi \cdot X>\varepsilon)>0$. Let $B$ be the set of $\mathbb{F}_{0}$-measurable random variables $b$ defined as follows:

$B=\left\{b\right.$ : there exists an $\mathbb{F}_{0}$-measurable $\xi$ such that $\|\xi\|_{\sim} \leq 1$ and $\xi \cdot X \geq b(\omega)$ a.s. $\}$

The set $B$ is nonempty, since $0 \in B$. Put

$$
l(\omega)=\operatorname{Ess} \sup B,
$$

where Ess sup means the essential supremum for a set of random variables (see $[\underline{6}$ for details).

Proposition 3.7. For all $\delta>0$, there exists an equivalent measure $\mathrm{P}^{*}$ with a bounded density and such that

$$
\left\|\mathrm{E}_{\mathrm{P}^{*}}\left[X / \mathbb{F}_{0}\right]\right\|_{\sim}^{*} \leq l(\omega)+\delta .
$$

Proof. The following result is similar to Lemma 3.6.

Lemma 3.8. Suppose that for no $\delta>0$ there is an $(\varepsilon+\delta) \sim$-arbitrage strategy for a random vector $Y \in \mathbb{R}^{d}$ with distribution $\mathrm{P}(d x)$; that is, the equality $\mathrm{P}\left(H_{\alpha \geq}^{\varepsilon+\delta}\right)=1$ implies that $\mathrm{P}\left(H_{\alpha>}^{\varepsilon+\delta}\right)=0$, where $\alpha \in \mathbb{R}^{d}$ is such that $\|\alpha\|_{\sim} \leq 1$. Then, for all $\delta>0$, there exists a positive function $g \in C\left(\overline{\mathbb{R}}^{d}\right)$ such that $\mathrm{E}[g(Y)]=1$ and $\|\mathrm{E}[Y g(Y)]\|_{\sim}^{*} \leq \varepsilon+\delta$.

The proof of Lemma 3.8 is rather simple. Since there exists a vector $\alpha$ such that $\|\alpha\|_{\sim} \leq 1$ and

$$
\mathrm{P}\left(H_{\alpha=}^{\varepsilon+\delta}\right)=1,
$$

we immediately conclude that the vector $\alpha$ generates an $(\varepsilon+\delta / 2) \sim$-arbitrage strategy.

Similarly to Theorem 3.5, we denote by $P(\omega, d x)$ the regular distribution of $X$ given $\mathbb{F}_{0}$ and introduce

$$
c(\omega)=\sup _{\|y\|_{\sim} \leq 1} \underset{x \in \mathbb{R}^{d}}{\operatorname{essinf}} x \cdot y,
$$

where the essential infimum is considered with respect to $P(\omega, \cdot)$. Then $c(\omega) \leq l(\omega)$. Indeed, consider the set

$$
\left\{(y, \omega):\|y\|_{1} \leq 1, x \cdot y \geq c(\omega)-\gamma(\bmod P(\omega, d x))\right\} \in \mathcal{B}\left(\mathbb{R}^{d}\right) \otimes \mathbb{F}_{0}
$$

for $\gamma>0$. By definition, the projection of this set onto $\Omega$ is of full measure, and thus the measurable selection theorem implies that there exists an $\mathbb{F}_{0}$-measurable vector $\xi(\omega)$ such that

$$
\xi(\omega) \cdot x \geq c(\omega)-\gamma \quad(\bmod P(\omega, d x))
$$

whence

$$
\xi(\omega) \cdot X(\omega) \geq c(\omega)-\gamma \quad \text { a.s. },
$$

that is, $(c(\omega)-\gamma) \in B$. Therefore $c(\omega)-\gamma \leq l(\omega)$ almost surely. Passing to the limit as $\gamma \rightarrow 0$, we prove the desired result.

Since there is no $(c(\omega)+\delta) \sim$-arbitrage strategy for almost all $\omega$ and for a random vector with distribution $P(\omega, d x)$, we follow the lines of the proof of Theorem 3.5 and construct a measurable function $G(\omega, x)$ such that

$$
\left\|\mathrm{E}\left[X(\omega) G(\omega, X(\omega)) / \mathbb{F}_{0}\right]\right\|_{\sim}^{*} \leq c(\omega)+\delta,
$$


then define $M(\omega)$ as before, and choose

$$
D=M G(\omega, X(\omega)) M(\omega)
$$

for $M=1 / \mathrm{E}[M(\omega)]$. Then the measure $P^{*}$ with density $D$ is as desired.

The following corollary of Proposition 3.7 is of its own interest.

Theorem 3.9. The following conditions are equivalent:

1. For no $\delta>0$ there is an $(\varepsilon+\delta) \sim$-arbitrage strategy for a single-period model.

2. For all $\delta>0$, there exists a measure $\mathrm{P}^{*} \sim \mathrm{P}$ with a bounded density such that

$$
\mathrm{E}_{\mathrm{P}^{*}}\left[\left\|\mathrm{E}_{P^{*}}\left[\triangle X / \mathbb{F}_{0}\right]\right\|_{\sim}^{*}\right] \leq \varepsilon+\delta .
$$

Proof. The proof is similar to that of Theorem 3.5 if one uses Proposition 3.7 .

\section{Fundamental theOREM FOR A MULTiPERIOD MODEL}

Put

$$
R_{T}^{\varepsilon}:=\left\{V_{T}: V_{T}=\sum_{t=1}^{T} \xi_{t} \cdot \Delta X_{t}-\varepsilon\right\},
$$

where $\xi$ is an arbitrary self-financing strategy bounded in the sense of relation (10), and where $\triangle X_{t}=X_{t}-X_{t-1}$. It is clear that $R_{T}^{\varepsilon}$ is the set of all possible terminal values which an investor can achieve if he starts with an initial capital $-\varepsilon$. Here $\xi$ is a strategy generated a particular terminal value.

Let $A_{T}^{\varepsilon}:=R_{T}^{\varepsilon}-L_{+}^{0}=\left\{V-V^{+}: V \in R_{T}^{\varepsilon}, V^{+} \in L_{+}^{0}\right\}$.

Denote by $\bar{A}$ the closure of a family $A$ of random variables in the sense of almost sure convergence.

Theorem 4.1. The following conditions are equivalent:

1. $R_{T}^{\varepsilon+\delta} \cap L_{+}^{0} \subset\{0\}$ for all $\delta>0$ (the property of no- $(\varepsilon+\delta)$-arbitrage).

2. $A_{T}^{\varepsilon+\delta} \cap L_{+}^{0} \subset\{0\}$ for all $\delta>0$.

3. $A_{T}^{\varepsilon+\delta} \cap L_{+}^{0} \subset\{0\}$ and $A_{T}^{\varepsilon+\delta}=\overline{A_{T}^{\varepsilon+\delta}}$ for all $\delta>0$.

4. $\overline{A_{T}^{\varepsilon+\delta}} \cap L_{+}^{0} \subset\{0\}$ for all $\delta>0$.

5. For all $\delta>0$, there exists $\mathrm{P}^{*} \sim \mathrm{P}$ such that

$$
\mathrm{E}_{\mathrm{P}^{*}}\left[\sum_{t=1}^{T}\left\|\mathrm{E}_{\mathrm{P}^{*}}\left[\triangle X_{t} / \mathbb{F}_{t-1}\right]\right\|_{\infty}\right] \leq \varepsilon+\delta,
$$

where $\triangle X_{t}:=X_{t}-X_{t-1}$ and where the Radon-Nikodym derivative is bounded, that is, $d \mathrm{P}^{*} / d \mathrm{P} \leq C$ for some $C>0$.

Remark 4.2. The assumptions of the classical no-arbitrage theorem yield that the corresponding intersections coincide with $\{0\}$. For example, the analogue of the above condition 1 in the classical theorem is $R_{T}^{0} \cap L_{+}^{0}=\{0\}$. Note that condition 1 means the same if applied to the classical case, since the trivial strategy leads to the zero terminal capital if the initial capital is zero. This, however, is not true for the initial capital $-\varepsilon$, for which a strategy with the zero terminal capital does not necessarily exist.

Proof. Our proof goes along the following scheme:

$$
\begin{aligned}
1 & \Leftrightarrow 5 \\
4 \Rightarrow & \mathbb{1} \\
2 & \Leftrightarrow 3 \Rightarrow 4
\end{aligned}
$$

The implications $[3 \Rightarrow 4],[4 \Rightarrow 2]$, and $[3 \Rightarrow 2]$ are obvious. 
Proof of the implication $[2 \Rightarrow 1]$. It is also obvious, since $R_{T}^{\varepsilon+\delta} \subset A_{T}^{\varepsilon+\delta}$ for all $\delta>0$ by definition.

Proof of the implication $[1 \Rightarrow 2]$. Assume that condition 1 holds for a fixed $\delta>0$, while condition 2 does not. This means that there exists $z \in A_{T}^{\varepsilon+\delta} \cap L_{+}^{0}$ such that $z \neq 0$. Then there are $\zeta \in R_{T}^{\varepsilon+\delta}$ and $u \in L_{+}^{0}$ such that $z=\zeta-u \geq 0$ implying $\zeta \geq u \geq 0 \Rightarrow \zeta \geq 0 \Rightarrow \zeta=0$, since condition 1 yields $R_{T}^{\varepsilon+\delta} \cap L_{+}^{0} \subset\{0\}$. Hence $\zeta$ can only be zero. Since $\zeta=0$, we conclude that $u=0$ as well, whence $z=0$. We obtained a contradiction, that is the implication $1 \Rightarrow 2$ is true, indeed.

Proof of the implication $[2 \Rightarrow 3]$. We prove the implication for an arbitrary $\delta>0$. We use the induction to prove that $A_{T}^{\varepsilon+\delta}$ is closed. First we justify the initial induction step; that is, we consider the case of $T=1$. Let

$$
H_{1}^{n} \triangle X_{1}-(\varepsilon+\delta)-r^{n} \rightarrow \zeta, \quad n \rightarrow \infty,
$$

almost surely where $H_{1}^{n}$ is an $\mathbb{F}_{0}$-measurable bounded $d$-dimensional random vector and $r^{n} \in L_{+}^{0}$. Thus $H_{1}^{n} \triangle X_{1}-(\varepsilon+\delta) \in R_{1}^{\varepsilon+\delta}$. We need to prove that there is a sequence of $\mathbb{F}_{0}$-measurable random vectors $\widetilde{H}_{1}^{k}$ converging almost surely and such that $\widetilde{r}^{k} \in L_{+}^{0}$ and $\widetilde{H}_{1}^{k} \triangle X_{1}-(\varepsilon+\delta)-\widetilde{r}^{k} \rightarrow \zeta$ as $k \rightarrow \infty$ almost surely.

Put $\underline{H_{1}}:=\lim _{n \rightarrow \infty} \inf _{k \leq n}\left\|H_{1}^{k}\right\|$. By the stochastic Bolzano-Weierstrass theorem [8], we can choose an $\mathbb{F}_{0}$-measurable $\widetilde{H}_{1}^{k}$ on the set $\Omega_{1}:=\left\{\underline{H_{1}}<\infty\right\}$ such that $\widetilde{H}_{1}^{k}(\omega)$ is a convergent subsequence of $H_{1}^{n}(\omega)$ for every $\omega$. Choosing $\bar{r}^{k}$ from the initial sequence for every $\widetilde{H}_{1}^{k}$, we complete the proof. This means that the proof follows if $\mathrm{P}\left\{\Omega_{1}\right\}=1$.

This is indeed the case, since $\mathrm{P}\left\{\Omega_{1}\right\}=\mathrm{P}\{\Omega\}=1$ (this follows from the boundedness of $\left.H_{1}^{n}\right)$. In fact, $\left\|H_{1}^{n}\right\|_{1}<1$ and this immediately implies that $\lim _{n \rightarrow \infty} \inf _{k \leq n}\left\|H_{1}^{k}\right\|<\infty$ almost surely.

Therefore the first induction step is justified. Assume that the implication $2 \Rightarrow 3$ holds for $T-1$ periods and $\sum_{t=1}^{T} H_{t}^{n} \triangle X_{t}-r^{n}-(\varepsilon+\delta) \rightarrow \zeta$ as $n \rightarrow \infty$ almost surely, where the vector $H_{t}^{n}$ is $\mathbb{F}_{t-1}$-measurable for every $t=1, \ldots, T$, bounded, and has dimension $d$. Moreover $r^{n} \in L_{+}^{0}$.

Following the same argument as in the case of $T=1$, we choose sequences $\widetilde{H}_{t}^{k}$ and $\widetilde{r}^{k}$ such that $\widetilde{H}_{1}^{k}$ converges almost surely. Namely, we first choose a subsequence of $\left(H_{1}^{n}\right)_{n=1}^{\infty}$ convergent almost surely and then find the corresponding convergent subsequences for other components. This reduces the problem for a model with $T$ periods to the same problem for a model with $T-1$ periods, which is solved, by the induction assumption. Indeed, solving the problem for a model with $T-1$ periods and choosing a sequence with almost surely convergent components such that $\sum_{t=2}^{T} \widetilde{H}_{t}^{k} \triangle X_{t}-\widetilde{r}^{k} \rightarrow \zeta-\lim _{k \rightarrow \infty} \widetilde{H}_{1}^{k}=: \widetilde{\zeta}$ almost surely, we can complete this sequence with an element $\widetilde{H}_{1}^{k}$ and obtain a desired sequence convergent almost surely.

Proof of the implication $[5 \Rightarrow 1]$. Consider an arbitrary fixed $\delta>0$. Since

$$
\sum_{t=1}^{T} \mathrm{E}_{\mathrm{P}^{*}}\left[\left\|\mathrm{E}_{\mathrm{P} *}\left[\triangle X_{t} / \mathbb{F}_{t-1}\right]\right\|_{\infty}\right] \leq \varepsilon+\delta,
$$

one can find a sequence $\left\{\varepsilon_{t}\right\}_{t=1}^{T}$ such that

$$
\sum_{t=1}^{T} \varepsilon_{t}=\varepsilon+\delta,
$$

where $\varepsilon_{t} \geq 0$ for all $t=1, \ldots, T$ and

$$
\mathrm{E}_{\mathrm{P}^{*}}\left[\left\|\mathrm{E}_{\mathrm{P}^{*}}\left[\triangle X_{t} / \mathbb{F}_{t-1}\right]\right\|_{\infty}\right] \leq \varepsilon_{t} \quad \text { for all } t=1, \ldots, T \text {. }
$$


Consider the corresponding single-period model for each period. Then

$$
\begin{aligned}
\mathrm{E}_{\mathrm{P}^{*}}\left[\left\|\mathrm{E}_{\mathrm{P}^{*}}\left[\triangle X_{t} / \mathbb{F}_{t-1}\right]\right\|_{\infty}\right] \leq \varepsilon_{t} & \Rightarrow \mathrm{E}_{\mathrm{P}^{*}}\left[\left\|\mathrm{E}_{\mathrm{P}^{*}}\left[\frac{S_{t}}{(1+r)^{t}}-\frac{S_{t-1}}{(1+r)^{t-1}} / \mathbb{F}_{t-1}\right]\right\|\right] \leq \varepsilon_{t} \\
& \Rightarrow \mathrm{E}_{\mathrm{P}^{*}}\left[\left\|\mathrm{E}_{\mathrm{P}^{*}}\left[\frac{S_{t}}{1+r}-S_{t-1} / \mathbb{F}_{t-1}\right]\right\|\right] \leq \varepsilon_{t}(1+r)^{t-1} .
\end{aligned}
$$

Applying Lemma 3.4 for each period, we prove that there is no $\varepsilon_{t}(1+r)^{t-1}$-arbitrage strategy for the period $t$.

Therefore, if an investor starts with the initial capital $-(\varepsilon+\delta)$, then he transforms the capital to $V_{1} \leq\left(-(\varepsilon+\delta)+\varepsilon_{1}\right)(1+r)$ after the first period. Otherwise we obtain a contradiction to Lemma 3.3 , since there is no $\varepsilon_{1}$-arbitrage strategy for the first period. We are allowed to drop the norm of $V_{1}$, since if a condition holds for the norm in $L_{\infty}$, then it holds for almost all $\omega \in \Omega$.

Similarly, after the second period, we have

$$
V_{2} \leq\left(V_{1}+\varepsilon_{2}(1+r)\right)(1+r)=\left(-(\varepsilon+\delta)+\varepsilon_{1}+\varepsilon_{2}\right)(1+r)^{2},
$$

since there is no $\varepsilon_{2}(1+r)$-arbitrage strategy for the second period. Continuing this reasoning for all further periods, we obtain that at the moment $T$,

$$
V_{T} \leq\left(-(\varepsilon+\delta)+\varepsilon_{1}+\cdots+\varepsilon_{T}\right)(1+r)^{T} .
$$

Now we use (6) and prove that the right hand side of (17) is zero. Hence

$$
V_{0}=-(\varepsilon+\delta) \text { and } V_{T} \leq 0
$$

for all $\omega \in \Omega$. The latter is the condition that there is no $(\varepsilon+\delta)$-arbitrage strategy for a multiperiod model. Since $\delta$ is arbitrary, the implication $5 \Rightarrow 1$ is proved.

Proof of the implication $[1 \Rightarrow 5]$. Assume that condition 1 holds, that is, there exists an $(\varepsilon+\delta)$-arbitrage strategy for each $\delta>0$.

Put $Y_{t}(\omega)=\triangle X_{t}(\omega)$ and define the set $B_{t}$ of $\mathbb{F}_{t}$-measurable random variables $b(\omega)$ as follows:

$$
\begin{array}{r}
B_{t}=\left\{b(\omega): \exists \text { predictable }\left\{\xi_{s}\right\}\right. \text { bounded in the sense of (1) } \\
\left.\qquad \text { and such that } \sum_{s=t+1}^{T} \xi_{s} \cdot Y_{s} \geq b(\omega)\right\} .
\end{array}
$$

We also define the essential supremum over $B_{t}$ and put

$$
l_{t}(\omega)=\operatorname{Ess} \sup B_{t}
$$

The measure is constructed step by step. Namely we construct a measure $\mathrm{P}_{t}^{*}$ for all $t$ such that it has a density, $\mathrm{P}_{t}^{*} \sim \mathrm{P}$, and

$$
\mathrm{E}_{\mathrm{P}_{t}^{*}}\left[\sum_{s=t}^{T}\left\|\mathrm{E}_{\mathrm{P}_{t}^{*}}\left[Y_{s} / \mathbb{F}_{s-1}\right]\right\| / \mathbb{F}_{t-1}\right] \leq l_{t-1}(\omega)+(1-(t-1) / T) \delta .
$$

The construction of the measure for $t=T$ is justified by Proposition 3.7. Assume that a measure $\mathrm{P}_{t+1}^{*}$ with the indicated properties has already been constructed. Consider the vector $Z_{t}=\left(Y_{t}, l_{t}(\omega)\right)$ and introduce the norm

$$
\|x\|_{\sim}=\max \left\{\sum_{i=1}^{d}\left|x^{i}\right|,\left|x^{d+1}\right|\right\}
$$


in the space $\mathbb{R}^{d+1}$. Now we define the family $B$ of $\mathbb{F}_{t-1}$-measurable random variables $b(\omega)$ as follows:

$$
\begin{aligned}
& B:=\left\{b(\omega): \exists \xi \in \mathbb{R}^{d+1} \text { that is } \mathbb{F}_{t-1}\right. \text {-measurable and such that } \\
& \left.\qquad\|\xi\|_{\sim} \leq 1 \text { and } \xi \cdot Z_{t} \geq b(\omega) \text { almost surely }\right\} .
\end{aligned}
$$

Let

$$
l^{\prime}(\omega)=\operatorname{Ess} \sup B .
$$

We prove that $l^{\prime}(\omega) \leq l_{t-1}(\omega)$ almost surely. Note that $B$ is a lattice, that is

$$
\max \left\{b^{\prime}, b^{\prime \prime}\right\} \in B
$$

for $b^{\prime}, b^{\prime \prime} \in B$. To prove the latter statement consider the vectors $\xi^{\prime}$ and $\xi^{\prime \prime}$ of the family $B$ and put

$$
\widetilde{\xi}=\xi^{\prime} \mathbb{1}_{\left\{b^{\prime}>b^{\prime \prime}\right\}}+\xi^{\prime \prime} \mathbb{1}_{\left\{b^{\prime} \leq b^{\prime \prime}\right\}} .
$$

The vector $\xi$ is $\mathbb{F}_{t}$-measurable, since $b^{\prime}$ and $b^{\prime \prime}$ are $\mathbb{F}_{t}$-measurable and moreover $\|\xi\|_{\sim} \leq 1$ almost surely. Thus there exists an increasing sequence $b_{n} \in B$ such that $b_{n} \rightarrow l^{\prime}(\omega)$ as $n \rightarrow \infty$ almost surely. The corresponding sequence of vectors is denoted by $\zeta(n)$. Similarly, there exists an increasing sequence $b_{n}^{\prime} \in B_{t}$ such that $b_{n}^{\prime} \rightarrow l_{t}(\omega)$ as $n \rightarrow \infty$ almost surely. Denote by $\varphi_{t}(n)$ the corresponding sequence of strategies. Put $\xi_{t}(n)=$ $\left(\zeta^{1}(n), \ldots, \zeta^{d}(n)\right)$ and $\xi_{s}(n)=\zeta^{d+1}(n) \varphi_{s}(n)$ for $s \geq t+1$. This strategy is predictable and bounded in the sense of (11). Moreover

$$
\liminf _{n \rightarrow \infty} \sum_{s=t}^{T} \xi_{s}(n) \cdot Y_{s} \geq l^{\prime}(\omega)
$$

whence $l^{\prime}(\omega) \leq l_{t-1}(\omega)$ almost surely.

Using Proposition 3.7 we construct a measure $\mathrm{P}_{t}^{*} \sim \mathrm{P}_{t+1}^{*}$ such that

$$
\left\|\mathrm{E}_{\mathrm{P}_{t}^{*}}\left[Z_{t} / \mathbb{F}_{t-1}\right]\right\|_{\sim}^{*} \leq l_{t-1}(\omega)+\delta / T \text {. }
$$

Then

$$
\begin{aligned}
&\left\|\mathrm{E}_{\mathrm{P}_{t}^{*}}\left[Z_{t} / \mathbb{F}_{t-1}\right]\right\|_{\sim}^{*}=\left\|\mathrm{E}_{\mathrm{P}_{t}^{*}}\left[Y_{t} / F_{t-1}\right]\right\|_{\infty}+\left|\mathrm{E}_{\mathbf{P}_{t}^{*}}\left[l_{t}(\omega) / \mathbb{F}_{t-1}\right]\right| \\
& \geq\left\|\mathrm{E}_{\mathbf{P}_{t}^{*}}\left[Y_{t} / F_{t-1}\right]\right\|_{\infty}+\left|\mathrm{E}_{\mathbf{P}_{t}^{*}}\left[\mathrm{E}_{\mathbf{P}_{t+1}^{*}}\left[\sum_{s=t+1}^{T}\left\|\mathrm{E}_{\mathbf{P}_{t+1}^{*}}\left[Y_{s} / \mathbb{F}_{s-1}\right]\right\| / \mathbb{F}_{t}\right] / \mathbb{F}_{t-1}\right]\right| \\
&-(1-(t+1) / T) \delta
\end{aligned}
$$

Putting

we obtain that for $s \geq t+1$,

$$
J(t, s)=\mathrm{E}_{\mathrm{P}_{t}^{*}}\left[\frac{d \mathrm{P}_{t+1}^{*}}{d \mathrm{P}_{t}^{*}} Y_{s} / \mathbb{F}_{s-1}\right] / \mathrm{E}_{\mathrm{P}_{t}^{*}}\left[\frac{d \mathrm{P}_{t+1}^{*}}{d \mathrm{P}_{t}^{*}} / \mathbb{F}_{s-1}\right],
$$

$$
\begin{aligned}
\mathrm{E}_{\mathrm{P}_{t}^{*}} & {\left[\mathrm{E}_{\mathrm{P}_{t+1}^{*}}\left[\left\|\mathrm{E}_{\mathrm{P}_{t+1}^{*}}\left[Y_{s} / \mathbb{F}_{s-1}\right]\right\|_{\infty} / \mathbb{F}_{t}\right] / \mathbb{F}_{t-1}\right] } \\
& =\mathrm{E}_{\mathrm{P}_{t}^{*}}\left[\mathrm{E}_{\mathrm{P}_{t}^{*}}\left[\frac{d \mathrm{P}_{t+1}^{*}}{d \mathrm{P}_{t}^{*}}\|J(t, s)\|_{\infty} / \mathbb{F}_{t}\right] / \mathrm{E}_{\mathrm{P}_{t}^{*}}\left[\frac{d \mathrm{P}_{t+1}^{*}}{d \mathrm{P}_{t}^{*}} / \mathbb{F}_{t}\right] / \mathbb{F}_{t-1}\right] \\
& =\mathrm{E}_{\mathrm{P}_{t}^{*}}\left[\left\|\mathrm{E}_{\mathrm{P}_{t}^{*}}\left[Y_{s} / \mathbb{F}_{s-1}\right]\right\|_{\infty} / \mathbb{F}_{t-1}\right] .
\end{aligned}
$$

The last equality holds, since $d \mathrm{P}_{t+1}^{*} / d \mathrm{P}_{t}^{*}$ is $\mathbb{F}_{t}$-measurable (the expectations are finite almost surely as the density is integrable). Substituting this result to (9), we prove (8) for the period $t$. It remains to note that inequality (8) for $t=1$ is equivalent to Theorem 4.1 (the boundedness of the resulting density follows from the boundedness of the density constructed for each period). 


\section{BIBLIOGRAPHY}

1. M. Harrison and S. Pliska, Martingales and stochastic integrals in the theory of continuous trading, Stochastic Process. Appl. 11 (1981), 215-260. MR622165 (83a:90022)

2. R. C. Dalang, A. Morton, and W. Willinger, Equivalent martingale measures and no-arbitrage in stochastic securities market models, Stochastics and Stochastics Reports 29 (1990), 185-201. MR.1041035 (91g:90056)

3. F. Delbaen and W. Schachermayer, A general version of the fundamental theorem of asset pricing, Mathematische Annalen 300 (1994), 463-520. MR1304434 (95m:90022b)

4. Yu. Kabanov and C. Stricker, Remarks on the True No-Arbitrage Property, Manuscript, Laboratoire de Mathématiques de Besançon, 2003.

5. A. N. Shiryaev, Essentials of Stochastic Finance. Facts, Models, Theory, "Fazis", Moscow, 1998; English transl., World Scientific, River Edge, NJ, 1999. MR.1695318 (2000e:91085)

6. H. Föllmer and A. Schied, Stochastic Finance. An Introduction in Discrete Time, 2nd edition, Walter de Gruyter, 2004. MR2169807 (2006d:91002)

7. Yu. S. Mishura, The fundamental theorem of financial mathematics for limited arbitrage, Applied Statistics. Actuarial and Financial Mathematics 2003, no. 1-2, 49-54. (Ukrainian)

8. Yu. M. Kabanov and Ch. Stricker, A teachers' note on no-arbitrage criteria, Séminaire de Probabilités XXXV, pp. 149-152, Lecture Notes in Math., 1755, Springer, Berlin, 2001, MR1837282 (2003c:60073)

9. Ch. Stricker, Arbitrage et lois de martingale, Ann. Inst. H. Poincaré Probab. Statist. 26 (1990), 451-460. MR 1066088 (91m:60080)

10. J. A. Yan, Caractérisation d'une classe d'ensembles convexes de $L_{1}$ ou $H_{1}$, Seminar on Probability, XIV (Paris, 1978/1979), pp. 220-222, Lecture Notes in Math., 784, Springer, Berlin, 1980. MR0580127(82c:60090)

Department of Probability Theory and Mathematical Statistics, Faculty for Mechanics and Mathematics, National Taras Shevchenko University, Academician Glushkov Avenue 6 , KYIV 03127, UKRAINE

E-mail address: myus@univ.kiev.ua

Department of Probability Theory and Mathematical Statistics, Faculty for Mechanics and Mathematics, National Taras Shevchenko University, Academician Glushkov Avenue 6 , KYIV 03127, UKRAINE

E-mail address: Pavlo.Shelyazhenko@gmail.com

Department of Probability Theory and Mathematical Statistics, Faculty for Mechanics and Mathematics, National Taras Shevchenko University, Academician Glushkov Avenue 6, KYIV 03127, UKRAINE

E-mail address: zhora@univ.kiev.ua

Received 29/JUL/2005

Translated by N. SEMENOV 